\title{
Altered microRNA expression in skeletal muscle results from high-fat diet-induced insulin resistance in mice
}

\author{
GUO-QING CHEN ${ }^{1}$, WEN-JING LIAN ${ }^{2}$, GUI-MING WANG ${ }^{2}$, \\ SONG WANG ${ }^{1}$, YUE-QIN YANG ${ }^{1}$ and ZHI-WEI ZHAO ${ }^{2}$

\begin{abstract}
${ }^{1}$ Department of Health Science, Wuhan Institute of Physical Education, Wuhan, Hubei; ${ }^{2}$ Department of Human Anatomy, West China School of Preclinical and Forensic Medicine, Sichuan University, Chengdu, Sichuan, P.R. China
\end{abstract}

Received January 16, 2012; Accepted February 23, 2012

DOI: $10.3892 / \mathrm{mmr} .2012 .824$

\begin{abstract}
Skeletal muscle insulin resistance induced by a high-fat diet has been implicated in the development of type 2 diabetes. However, the precise molecular mechanisms involved are only partially understood. Recently, studies have shown that microRNAs play an important role in insulin resistance in various tissues. In this study, microRNA expression profiles of skeletal muscle of mice fed a high-fat or normal diet were analyzed using microarrays and the results were confirmed by real-time reverse-transcription polymerase chain reaction. Gene Ontology (GO) and pathway mapping tools were employed to analyze systemically the biological processes and signaling pathways affected by the differential expression of microRNAs. In this study, we show that 30 microRNAs are differentially expressed between 2 groups of mice. Compared to the mice fed a normal diet, there were 8 microRNAs up-regulated and 22 microRNAs down-regulated in the highfat diet-fed mice. Furthermore, we confirm that the MAPK signaling pathway highlighted in this study is involved in skeletal muscle insulin resistance. These results indicate that skeletal muscle insulin resistance induced by a high-fat diet is associated with a group of microRNAs. GO and pathway mapping are a valid and effective approach for analyzing the function of microRNAs and the results could be a guideline for further investigation.
\end{abstract}

\section{Introduction}

Over the past several decades, the prevalence of type 2 diabetes and obesity has increased dramatically due to western style diets and sedentary lifestyles. Excess dietary fat plays

Correspondence to: Dr Zhi-Wei Zhao, Department of Human Anatomy, West China School of Preclinical and Forensic Medicine, Sichuan University, No. 17 Renmin South Road, Chengdu, Sichuan 610041, P.R. China

E-mail: zhiwzhao@163.com

Key words: microRNA, insulin resistance, high-fat diet, skeletal muscle, pathway analysis a crucial role and is a determinant of the current epidemic of insulin resistance. There have been an increasing number of studies which confirm that lipid accumulation in the skeletal muscle induces insulin resistance in various groups of subjects, including type 2 diabetes and obesity patients (1). Skeletal muscle, which makes up $40 \%$ of the body mass in humans and other mammalian species, is the predominant tissue for insulin-stimulated glucose and lipid utilization under physiological conditions. In a state of insulin resistance, however, glucose uptake and utilization in skeletal muscle are dramatically decreased; at the same time, fatty acid uptake is increased, but the capacity for fat oxidation is reduced $(2,3)$. Although it has been known that incomplete fatty acid oxidation and mitochondrial overload lead to insulin resistance $(4,5)$, the underlying molecular mechanisms of skeletal muscle insulin resistance are only partially understood.

microRNAs are a class of endogenous non-coding RNA molecules that negatively regulate gene expression. microRNAs bind to the 3'-untranslated region of target mRNAs leading to mRNA cleavage or translation inhibition. It has also been shown that microRNAs target more than $30 \%$ of protein-coding genes (6). In mammals, microRNAs have been implicated in the modulation of various biological processes, including organ development, cell proliferation and differentiation, apoptosis and response to stresses (7). Growing evidence has also indicated that microRNAs are involved in the pathogenesis of cancer, cardiovascular diseases, obesity and diabetes (8-11). In particular, in obesity and diabetes, it has been well demonstrated that microRNAs, such as miR-375, miR-29, miR-320, miR-103/107 and miR-126, play a crucial role in the regulation of glucose and lipid metabolism through the control of pancreatic islet cell function, adipocyte insulin resistance and hepatocyte insulin signaling and glucose homeostasis (12-16). As for skeletal muscle, microRNAs also play a critical role in muscle development, function, disorders and regeneration. For example, miR-1 promotes myoblast differentiation via the inhibition of histone deacetylase 4 (HDAC4) (17); miR-133a stimulates myoblast proliferation through the repression of serum response factor (SRF) (17); miR-206 influences the differentiation program of myoblasts via an indirect down-regulation of the helixloop-helix Id protein (18); miR-27 promotes myogenesis by down-regulating Pax3 (19). All these indicate that microRNAs are essential for the development and function of skeletal muscle 
and are considered to be a novel therapeutic target. However, the expression and role of microRNAs in skeletal muscle insulin resistance is not yet well established, although several studies have suggested that microRNAs are involved in skeletal muscle insulin resistance in animal models or type 2 diabetes patients (13,20-23). Therefore, in our study we established a skeletal muscle insulin resistance mouse model (mice were fed a high-fat diet) and sought to determine the expression profiles of microRNAs by microRNA microarray and examine the biological processes and signaling pathways involved in skeletal muscle insulin resistance by Gene Ontology (GO) and pathway mapping tools.

\section{Materials and methods}

Animals. Forty male, 3-week-old wild-type C57BL/6 mice (body weight, $15.41 \pm 2.06 \mathrm{~g}$ ) were maintained at room temperature on a 12-h light-dark cycle, with free access to chow and water. The animals were randomly divided into 2 groups $(n=20)$ : the normal diet (ND) group and the high-fat diet (HFD) group (58\% fat, $25 \%$ protein and $17 \%$ carbohydrate, as a percentage of total kcal) for 12 weeks. At the end of the 12-week feeding period, all mice were fasted for $12 \mathrm{~h}$ and blood samples were collected for fasting blood glucose, plasma insulin and lipid assay. In each group, animals were further divided into 2 subgroups of 10 animals each for glucose tolerance testing (GTT) and insulin tolerance testing (ITT). For GTT, mice were given an intraperitoneal injection of glucose $(2 \mathrm{~g} / \mathrm{kg}$ body weight), blood samples were collected from the tail vein immediately before and 15, 30, 60, 90 and $120 \mathrm{~min}$ after glucose administration, and blood glucose levels were measured with o-toluidine reagent (Sigma). For ITT, the animals were fasted for $4 \mathrm{~h}$ prior to an intraperitoneal injection of insulin $(1 \mathrm{U} / \mathrm{kg}$ body weight). Blood samples were withdrawn from tail vein at 0 , 15, 30, 60 and 120 min post-injection. Finally, all animals were sacrificed with a high dose of sodium thiopentone $(100 \mathrm{mg} / \mathrm{kg}$ body weight). The gastrocnemii were carefully dissected from the surrounding tissue, frozen in liquid nitrogen and stored at $-80^{\circ} \mathrm{C}$ until further analysis. The study was approved by the Institutional Animal Ethics Committee of Sichuan University.

Isolation of microRNA. Total RNA was isolated using TRIzol reagent (Invitrogen) according to the manufacturer's instructions. The concentration and quality of total RNA were measured by the UV absorbance at 260 and $280 \mathrm{~nm}$ (260/280 nm) and examined by gel electrophoresis. microRNA was isolated from the total RNA using the mirVana ${ }^{\mathrm{TM}}$ miRNA isolation kit (Ambion) according to the manufacturer's instructions.

microRNA microarray. microRNA was labeled using the miRCURY ${ }^{\mathrm{TM}}$ Array Labelling kit (Exiqon). The $\mathrm{Hy} 3^{\mathrm{TM}}$ fluorescent label was used for dyeing. Then, the labeled samples were concentrated by the RNeasy mini kit (Qiagen) according to the manufacturer's instructions and hybridized on the miRCURY ${ }^{\mathrm{TM}}$ LNA microRNA Arrays (v.10.0; Exiqon, Denmark). Three microarray chips were analyzed for each of the 2 groups ( 6 chips in total) representing 1 animal per chip. Labeling efficiency was evaluated by analyzing the signals from control spike-in capture probes. A 635-nm laser was used to scan the slide using Genepix 400B and data were analyzed by Genepix Pro 6.0.

Real-time polymerase chain reaction (PCR) quantification of microRNA. RNA was reverse-transcribed to cDNA with gene-specific primers according to the TaqMan MicroRNA Assay protocol (PE Applied Biosystems). Real-time PCR was performed using Mastercycler ep Realplex (Eppendorf). The 20- $\mu 1$ PCR included $1 \mu 1$ reverse transcription product, 2X TaqMan Universal PCR master mix and $2 \mu \mathrm{l}$ of primers and was performed for $15 \mathrm{sec}$ at $95^{\circ} \mathrm{C}$ and $1 \mathrm{~min}$ at $60^{\circ} \mathrm{C}$ for 40 cycles followed by the thermal denaturation protocol. U6 small nuclear RNA was used as the internal control to normalize RNA input in the real-time PCR assay. The expression of microRNA relative to U6 RNA was determined using the $2^{-\triangle \Delta C T}$ method (24). All experiments were carried out in triplicates and repeated 3 times.

Western blotting. For protein extraction, mice gastrocnemii were ground into powder in liquid nitrogen with a pre-cooled mortar and pestle. The tissue powder was homogenized in $500 \mu \mathrm{l}$ of lysis buffer [50 mmol/1 Tris- $\mathrm{HCl}$ (pH 7.4), 1\% NP-40, $0.25 \%$ sodium deoxycholate, $150 \mathrm{mmol} / 1 \mathrm{NaCl}, 1 \mathrm{mmol} / 1$ EDTA, $1 \mathrm{mmol} / 1$ phenylmethylsulfonyl fluoride, $1 \mathrm{mmol} / 1$ $\mathrm{Na}_{3} \mathrm{VO}_{4}$ and $\left.1 \mathrm{mmol} / 1 \mathrm{NaF}\right]$ and incubated at $4^{\circ} \mathrm{C}$ for $30 \mathrm{~min}$, followed by centrifugation at $10,000 \mathrm{x} \mathrm{g}$ at $4^{\circ} \mathrm{C}$ for $20 \mathrm{~min}$. The lysates were collected and the protein concentration was determined using the BCA Protein Assay kit (Merck). Equal amounts of protein were separated by SDS-PAGE and transferred to polyvinylidene difluoride membranes (GE Healthcare Biosciences). The membranes were incubated with primary antibodies against ERK1/2, JNK, P38 and p-ERK1/2, p-JNK, p-P38 (1:1,000; Santa Cruz Biotechnology). Antibody binding was revealed by incubation with horseradish peroxidase-conjugated secondary antibodies (Santa Cruz Biotechnology) and an ECL Plus immunoblotting detection system (GE Healthcare Biosciences). Signals were quantified using NIH ImageJ 1.63 Software.

microRNA data analysis. Signal intensities for each spot were analyzed and calculated by the ImageQuant 5.0 (Amersham Pharmacia Biotech, Ltd.) and Array vision 6.0 (Imaging Research, Ltd.). Signal intensities for each spot were scanned and calculated by subtracting local background (based on the median intensity of the area surrounding each spot) from total intensities. An average value of the three spot replicates of each microRNA was generated after data transformation (to convert any negative value to 0.01 ), normalization was performed using the per-chip 50th percentile method that normalizes each chip on its median, allowing the comparison among chips. To highlight microRNAs that characterized each group, a per-gene on median normalization was performed, which normalizes the expression of every microRNA on its median among samples.

Potential microRNA target genes were identified by miRDB (http://mirdb.org/miRDB/) (25). GO enrichment and pathway mapping of the target genes was performed using the web-based tool, DAVID (http://david.abcc.ncifcrf.gov/) (26). Using DAVID software, Fisher's exact $\mathrm{p}$-value $(\mathrm{p}<0.05)$ was calculated to judge whether the observed difference was significant. 
Table I. Body weight and blood glucose, plasma insulin and lipid levels in the 2 groups of mice.

\begin{tabular}{lrc}
\hline & ND & HFD \\
\hline Body weight $(\mathrm{g})$ & $22.45 \pm 2.27$ & $36.14 \pm 2.03^{\mathrm{a}}$ \\
Serum insulin $(\mathrm{ng} / \mathrm{ml})$ & $1.17 \pm 0.08$ & $3.31 \pm 0.16^{\mathrm{a}}$ \\
Blood glucose $(\mathrm{mg} / \mathrm{dl})$ & $117.12 \pm 7.51$ & $142 \pm 6.02$ \\
FFAs $(\mathrm{mmol} / \mathrm{l})$ & $1.09 \pm 0.10$ & $1.38 \pm 0.11^{\mathrm{a}}$ \\
TC $(\mathrm{mmol} / \mathrm{l})$ & $2.16 \pm 0.27$ & $3.84 \pm 0.32^{\mathrm{a}}$ \\
TG $(\mathrm{mmol} / \mathrm{l})$ & $0.54 \pm 0.06$ & $1.07 \pm 0.13^{\mathrm{a}}$ \\
\hline
\end{tabular}

Data represent the means \pm SD. ${ }^{a} p<0.01$ vs. the normal diet (ND) group. HFD, high-fat diet group; FFAs, free fatty acids; TC, total cholesterol; TG, triglyceride.

\section{Results}

High-fat high-sugar diet induces obesity and insulin resistance. The characteristics of HFD and ND mice are shown in Table I. The high-fat diet induced an increase in body weight, blood glucose, plasma insulin and lipid levels. After 12 weeks of high-fat diet, the fasting blood glucose levels increased in the HFD compared to the ND mice. Investigation of the insulin sensitivity of the ND and HFD mice by insulin tolerance testing revealed that the glucose-lowering effect of insulin was significantly impaired in the HFD mice compared to the control mice (Fig. 1B). In addition, the glucose tolerance testing showed significantly higher levels of blood glucose in the HFD mice compared to the ND mice (Fig. 1A). Therefore, the HFD mice exhibited insulin resistance.

microRNA expression profiling in HFD mice and ND mice skeletal muscle. To assess whether the microRNA expression was affected in the HFD mice skeletal muscle, we examined the expression profiles of microRNAs in the gastrocnemii of the HFD and control mice. Following array processing and normalization of raw array data, 30 mouse microRNAs were differentially expressed between the 2 groups of mice, in which 22 microRNAs were down-regulated and 8 microRNAs were up-regulated in HDF mice (Fig. 2, Table II). To assess the validity of the microarray data, real-time quantitative PCR was performed to compare microRNA expression levels between the 2 groups of mice. Seven microRNAs (miR-1, miR-133a, miR-206, miR-23b, miR-143, miR-125a and miR-106b) were selected at random from the array dataset to validate the microarray data. In all cases, real-time PCR data were consistent with the results of the microarray, suggesting that the data set obtained from the microarray analysis accurately reflected microRNA expression differences between the HFD and ND mice (Fig. 3).

Target gene predictions and $G O$ and pathway mapping analysis. To identify the biological pathways overrepresented among the predicted targets of the 30 differentially expressed microRNAs, we performed a pathway enrichment analysis using the web-based application, miRDB. We then employed the GO approach using the DAVID tool as an unbiased method for identifying the predominant biological processes and path-
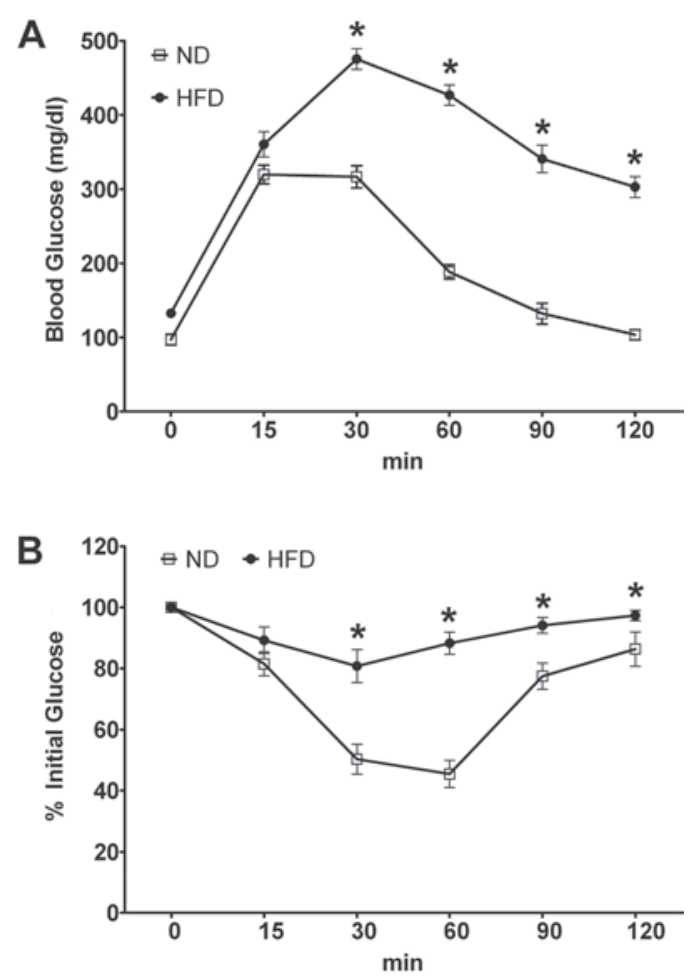

Figure 1. Curve of glucose tolerance and insulin tolerance tests. (A) Glucose tolerance testing; (B) insulin tolerance testing. ${ }^{*} \mathrm{p}<0.01$ vs. the normal diet (ND) group. HFD, high-fat diet group.
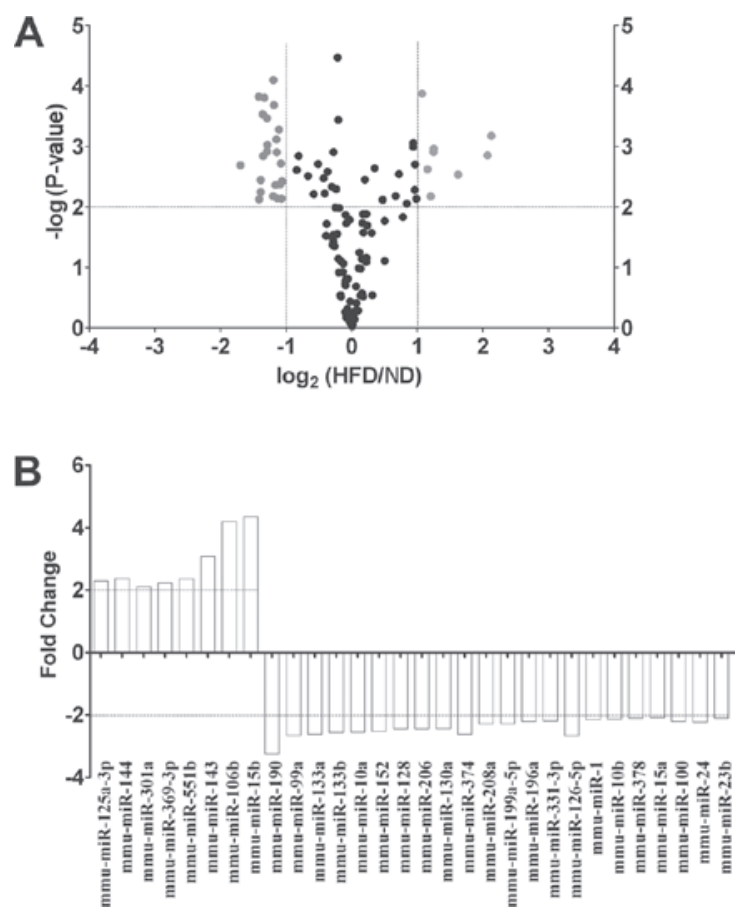

Figure 2. Differential expression microRNAs between control mice (ND) and high-fat diet (HFD) mice. (A) Volcano plots showing the change in microRNA expression between the 2 groups of mice. (B) Fold change in the expression of significantly altered microRNAs (fold change $\geq 2$ and $\mathrm{p}<0.01$ ) in skeletal muscle after 12 weeks of HFD.

ways representing the target genes. The results showed that there was a broad range of biological processes associated with the target genes. The main groups of genes were associated 
Table II. Significantly dysregulated microRNAs between the 2 groups of mice.

\begin{tabular}{|c|c|c|c|c|c|c|}
\hline \multirow[t]{2}{*}{ microRNAs } & \multicolumn{2}{|c|}{ ND NFI } & \multicolumn{2}{|c|}{ HFD NFI } & \multirow[t]{2}{*}{ Fold change } & \multirow[t]{2}{*}{$\mathrm{p}$-value } \\
\hline & Mean & SD & Mean & SD & & \\
\hline \multicolumn{7}{|l|}{ Up-regulated } \\
\hline mmu-miR-125a-3p & $2,003.75$ & 1.66 & $4,611.61$ & 370.52 & 2.30 & 6.69E-03 \\
\hline mmu-miR-144 & $2,858.35$ & 56.13 & $6,796.53$ & 601.76 & 2.38 & $1.09 \mathrm{E}-03$ \\
\hline mmu-miR-301a & 175.96 & 3.50 & 369.89 & 2.51 & 2.10 & $1.34 \mathrm{E}-04$ \\
\hline mmu-miR-369-3p & 77.15 & 4.58 & 171.58 & 4.40 & 2.22 & 2.39E-03 \\
\hline mmu-miR-551b & 394.14 & 20.63 & 934.83 & 40.36 & 2.37 & $1.24 \mathrm{E}-03$ \\
\hline mmu-miR-143 & $6,793.33$ & $1,105.43$ & $20,878.58$ & 413.42 & 3.07 & 2.93E-03 \\
\hline mmu-miR-106b & $1,302.53$ & 81.47 & $5,460.72$ & 233.00 & 4.19 & $1.40 \mathrm{E}-03$ \\
\hline mmu-miR-15b & $1,068.61$ & 118.04 & $4,662.89$ & 64.73 & 4.36 & $6.71 \mathrm{E}-04$ \\
\hline \multicolumn{7}{|l|}{ Down-regulated } \\
\hline mmu-miR-190 & 660.83 & 25.90 & 204.00 & 22.07 & -3.24 & $2.05 \mathrm{E}-03$ \\
\hline mmu-miR-99a & 610.75 & 35.25 & 229.36 & 22.78 & -2.66 & $7.53 \mathrm{E}-03$ \\
\hline mmu-miR-133a & $4,057.91$ & 233.84 & $1,551.72$ & 345.96 & -2.62 & $5.71 \mathrm{E}-03$ \\
\hline mmu-miR-133b & 844.09 & 23.82 & 329.36 & 22.78 & -2.56 & 2.95E-04 \\
\hline mmu-miR-10a & 849.24 & 20.53 & 332.99 & 47.08 & -2.55 & $1.44 \mathrm{E}-03$ \\
\hline mmu-miR-152 & $2,530.07$ & 48.37 & $1,004.17$ & 54.99 & -2.52 & $1.58 \mathrm{E}-04$ \\
\hline mmu-miR-128 & 760.78 & 14.69 & 310.57 & 9.96 & -2.45 & $3.43 \mathrm{E}-04$ \\
\hline mmu-miR-206 & 945.46 & 19.37 & 386.41 & 43.53 & -2.45 & $1.22 \mathrm{E}-03$ \\
\hline mmu-miR-130a & $2,530.07$ & 48.37 & $1,037.50$ & 112.73 & -2.44 & 9.43E-04 \\
\hline mmu-miR-374 & 658.97 & 31.28 & 251.92 & 39.57 & -2.62 & $3.60 \mathrm{E}-03$ \\
\hline mmu-miR-208a & $1,703.17$ & 35.98 & 743.55 & 29.78 & -2.29 & $8.01 \mathrm{E}-05$ \\
\hline mmu-miR-199a-5p & $1,749.50$ & 32.69 & 767.86 & 21.70 & -2.28 & $2.08 \mathrm{E}-04$ \\
\hline mmu-miR-196a & 946.53 & 29.36 & 427.39 & 23.64 & -2.21 & 7.69E-04 \\
\hline mmu-miR-331-3p & $1,444.78$ & 133.93 & 657.16 & 31.86 & -2.20 & 7.22E-03 \\
\hline mmu-miR-126-5p & $2,905.86$ & 60.36 & $1,086.53$ & 22.83 & -2.67 & $1.51 \mathrm{E}-04$ \\
\hline mmu-miR-1 & $1,545.04$ & 34.28 & 716.53 & 4.91 & -2.16 & $5.29 \mathrm{E}-04$ \\
\hline mmu-miR-10b & $1,748.44$ & 16.24 & 822.36 & 88.75 & -2.13 & 4.25E-03 \\
\hline mmu-miR-378 & $1,049.36$ & 61.12 & 500.10 & 37.60 & -2.10 & 7.31E-03 \\
\hline mmu-miR-15a & $3,455.00$ & 161.87 & $1,654.25$ & 35.68 & -2.09 & 3.79E-03 \\
\hline mmu-miR-100 & $1,175.57$ & 53.69 & 532.28 & 15.21 & -2.21 & $1.25 \mathrm{E}-03$ \\
\hline mmu-miR-24 & 945.46 & 19.37 & 423.07 & 78.35 & -2.23 & 4.37E-03 \\
\hline mmu-miR-23b & 845.46 & 19.37 & 403.07 & 78.35 & -2.10 & $6.66 \mathrm{E}-03$ \\
\hline
\end{tabular}

NFI, normalized fluorescence intensity with background subtracted. HFD/ND ratio $>2.0$ or $<0.5 ; \mathrm{p}<0.01$ is significant. ND, normal diet; HFD, high-fat diet; SD, standard deviation.

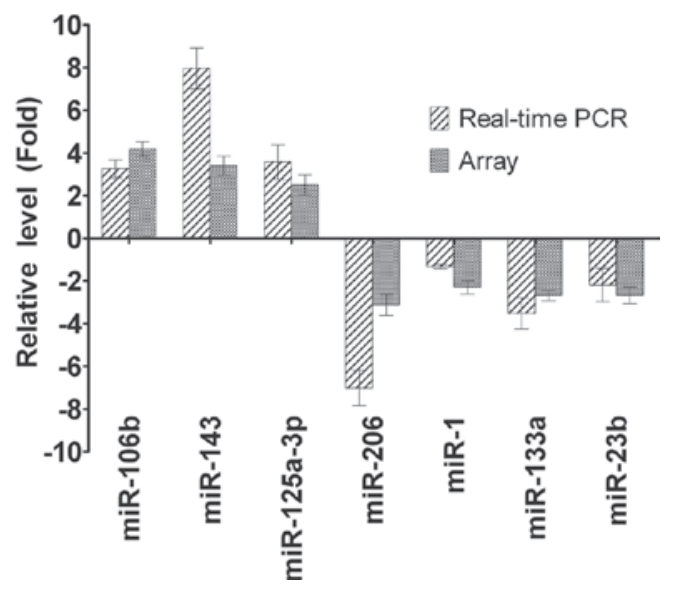

Figure 3. Validation of microarray data using real-time RT-PCR. with the cellular metabolic process, regulation of transcription, intracellular signaling cascade and cell death (Table III).

We then used the pathway mapping tools to identify the biological pathways of the target genes. The significantly overrepresented KEGG pathways are listed in Table IV.

Expression of MAPK signaling pathway. As described in the results of GO and pathway mapping, the MAPK signaling pathway may be involved in skeletal muscle insulin resistance induced by HFD. In order to validate this result, we detected the expression of ERK1/2, JNK and P38 in the gastrocnemii in HFD and control mice by western blotting. The results showed that HFD caused a significant up-regulation of p-JNK and p-P38, but not of p-ERK1/2 in the gastrocnemii (Fig. 4). 
Table III. Gene Ontology (GO) of differentially expressed microRNAs.

\begin{tabular}{lcc}
\hline Term & p-value & Fold enrichment \\
\hline GO:0031323 - regulation of cellular metabolic process & $4.48 \mathrm{E}-15$ & 1.46 \\
GO:0045449 - regulation of transcription & $1.16 \mathrm{E}-13$ & 1.50 \\
GO:0009966 - regulation of signal transduction & $1.04 \mathrm{E}-03$ & 1.43 \\
GO:0006511 - ubiquitin-dependent protein catabolic process & $3.70 \mathrm{E}-03$ & 1.92 \\
GO:0043067 - regulation of programmed cell death & $5.50 \mathrm{E}-03$ & 1.39 \\
GO:0051726 - regulation of cell cycle & $7.75 \mathrm{E}-03$ & 1.65 \\
GO:0007411 - axon guidance & $7.95 \mathrm{E}-03$ & 2.05 \\
GO:0016567 - protein ubiquitination & $8.73 \mathrm{E}-03$ & 2.32 \\
GO:0043161 - proteasomal ubiquitin-dependent protein catabolic process & $1.10 \mathrm{E}-02$ & 3.15 \\
GO:0000165 - MAPKKK cascade & $3.06 \mathrm{E}-02$ & 1.76 \\
\hline
\end{tabular}

Table IV. Over-represented KEGG pathways associated with differentially expressed microRNAs.

\begin{tabular}{llc}
\hline KEGG pathway term & p-value & Fold enrichment \\
\hline mmu04360: Axon guidance & $2.46 \mathrm{E}-06$ & 2.71 \\
mmu04010: MAPK signaling pathway & $1.81 \mathrm{E}-04$ & 1.86 \\
mmu05200: Pathways in cancer & $1.32 \mathrm{E}-03$ & 1.65 \\
mmu04144: Endocytosis & $2.14 \mathrm{E}-03$ & 1.82 \\
mmu04114: Oocyte meiosis & $3.55 \mathrm{E}-03$ & 2.09 \\
mmu04120: Ubiquitin mediated proteolysis & $4.55 \mathrm{E}-03$ & 1.96 \\
mmu04660: T cell receptor signaling pathway & $4.70 \mathrm{E}-03$ & 2.04 \\
mmu04310: Wnt signaling pathway & $1.24 \mathrm{E}-02$ & 1.79 \\
mmu04722: Neurotrophin signaling pathway & $1.28 \mathrm{E}-02$ & 1.85 \\
mmu00310: Lysine degradation & $1.31 \mathrm{E}-02$ & 2.78 \\
mmu04960: Aldosterone-regulated sodium reabsorption & $1.52 \mathrm{E}-02$ & 2.71 \\
mmu00534: Heparan sulfate biosynthesis & $1.65 \mathrm{E}-02$ & 3.28 \\
mmu04910: Insulin signaling pathway & $2.05 \mathrm{E}-02$ & 1.94 \\
mmu04340: Hedgehog signaling pathway & $2.37 \mathrm{E}-02$ & 2.35 \\
mmu04664: Fc $\varepsilon$ RI signaling pathway & $2.59 \mathrm{E}-02$ & 2.01 \\
mmu00511: Other glycan degradation & $3.23 \mathrm{E}-02$ & 3.96 \\
mmu04370: VEGF signaling pathway & $3.45 \mathrm{E}-02$ & 2.00 \\
mmu05210: Colorectal cancer & $3.60 \mathrm{E}-02$ & 1.91 \\
mmu04350: TGF- $\beta$ signaling pathway & $3.89 \mathrm{E}-02$ & 1.89 \\
\hline
\end{tabular}

KEGG pathway mapping was performed using the DAVID tool. Shown are the KEGG pathways that are significantly over-represented in the targets of differentially expressed microRNAs between the high-fat diet- and normal diet-fed mice ( $\mathrm{p}<0.05$ and fold change $>1.5)$.

\section{Discussion}

Skeletal muscle insulin resistance is the precondition for the onset of type 2 diabetes. For many years, it has been known that excess lipids accumulate in the skeletal muscle and disrupt a number of signaling pathways, leading to insulin resistance. The precise molecular mechanisms linking lipid oversupply to defective insulin action are not yet well understood. Increasing reports have described the critical role of microRNAs in diabetes and skeletal muscle disease. A number of microRNAs, miR-1, miR-133a, miR-133b and miR-206, the so-called myomiRs, have been shown to control muscle development and function through their regulation of key genes, such as MyoD, myogenin, myocyte enhancer factor 2 and myocardin-related transcription factor-A, controlling myogenesis $(17,18)$. It has also been suggested that microRNAs are involved in the regulation of metabolism, insulin secretion and pancreatic $\beta$-cell development (12-16). However, their role in skeletal muscle insulin resistance is not yet well established. To date, only few studies have investigated the effects of insulin resistance on microRNA expression in skeletal muscle $(13,20-23)$. In the present study, we investigated the insulin resistance in skeletal muscle induced by HFD at microRNA levels and analyzed the relevant pathways of differentially expressed microRNAs, in order to provide a more comprehensive understanding of the molecular network of the insulin resistance in skeletal muscle. 

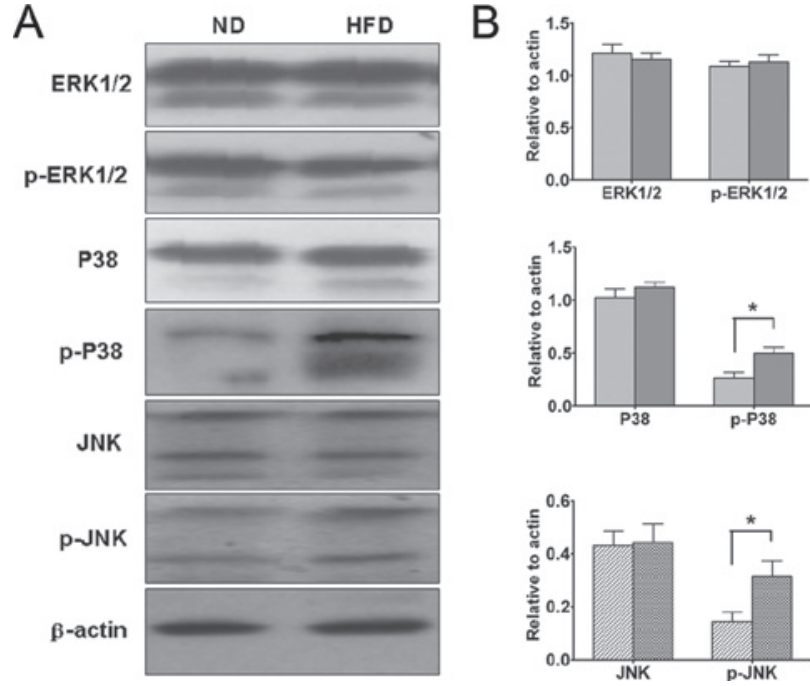

DNFD

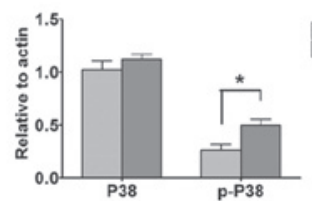

RHF

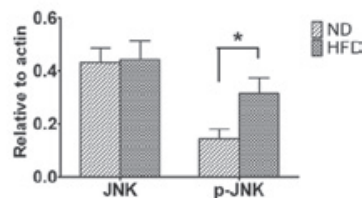

Figure 4. Expression changes in MAPK signaling protein phosphorylation of mice gastrocnemii due to high-fat diet shown by western blotting. * $\mathrm{p}<0.05 \mathrm{vs}$. the normal diet (ND) group. HFD, high-fat diet group.

To our knowledge, no reports are available on the characterization of microRNAs involved in insulin resistance in skeletal muscle induced by HFD.

Therefore, using a microarray platform, we compared the microRNA expressions in ND- and HFD-fed mice and identified 30 differentially expressed microRNAs. Among them, miR-1, miR-133 and miR-206 have been previously linked to skeletal muscle disease or function, and have been thought to act as the key regulators of myogenesis and function $(17,18,27,28)$. In our study, all 3 microRNAs were down-regulated in HFD-fed mice; these may be relevant to decreased myogenic response in insulin resistance in skeletal muscle (29). Among these differentially expressed microRNAs, several (such as mmu-miR-144, mmu-miR-10b, mmu-miR-451, mmu-miR133a and mmu-miR-99a) are well characterized and have been previously implicated in skeletal muscle in various animal models of diabetes or diabetes patients $(13,20-23)$. Notably, the direction of microRNA expressional change (up- or downregulation), however, differs between each diabetic animal model. In particular, miR-10b was down-regulated in our data, and has also been shown to be down-regulated in GK rats and type 2 diabetes patients (13,20-22); however, it has been shown to be up-regulated in HFD and streptozotocin (STZ)treated rats (23); miR-99a and miR-100 were down-regulated in our high-fat diet model; however, they have been shown to be up-regulated in HFD and STZ-treated rats (23). These results indicate that skeletal muscle insulin resistance induced by various methods may involve different mechanisms, thus suggesting that different therapeutic strategies are required.

In order to gain further information into the underlying molecular mechanisms of the microRNA involvement in insulin resistance of skeletal muscle, we predicted the targets of differentially expressed microRNAs, and then analyzed the gene annotation and signaling pathway enrichment. The results revealed that the microRNA targets are involved in a series of biological processes, cellular metabolic processes and regulation of transcription, intracellular signaling cascade and cell death, which indicates that insulin resistance induced by HFD involves a complex genetic cellular response. As for the signaling pathway mapping, we found 19 significant signaling pathways relevant to insulin resistance in skeletal muscle. Among these signaling pathways, important signal transduction pathways related to cell proliferation (MAPK, Wnt, neurotrophin, hedgehog, VEGF and TGF- $\beta$ ), metabolism (Lysine degradation, heparin sulfate biosyntheses and other glycan degradation), protein degradation (ubiquitin mediated proteolysis) and oncogenic (colorectal cancer) signaling pathways were enriched. Some of them have already been reported to be involved in insulin resistance. For example, MAPK and the insulin signaling pathway have been confirmed to be involved in skeletal muscle or other tissue insulin resistance in various animal models (30,31). Our western blotting results also showed that the key proteins of the MAPK signaling pathway, such as p38 MAPK and p-JUK, were up-regulated in our model. It is interesting that the signaling pathway axon guidance, neurotrophin signaling pathway and notch signaling pathway were also involved in insulin resistance in our pathway analysis results. Functional identity revealed by target prediction and pathway mapping confirmed that microRNA regulated the skeletal muscle insulin resistance by multiple signaling pathways, although further study is required to validate the differentially expressed microRNA-targeted genes involved in the above-mentioned signaling pathways.

In conclusion, the results presented in this study support the hypothesis that microRNAs play a mediatory role in insulin resistance in skeletal muscle induced by HFD. A 12-week HFD induced the differential expression of a set of microRNAs in skeletal muscle, which in turn regulated the expression of target genes involved in a series of biological processes and signaling pathways. Although the possible mechanism of how HFD regulates microRNA expression remains unclear, our study provides some interesting results for insulin resistance induced by a HFD. This information may be helpful for future investigations into the mechanisms and pathology of skeletal muscle insulin resistance.

\section{Acknowledgements}

This study was supported by the Research Foundation of the Education Department of Hubei Province, China (grant no. Q20104101).

\section{References}

1. Hegarty BD, Furler SM, Ye J, Cooney GJ and Kraegen EW: The role of intramuscular lipid in insulin resistance. Acta Physiol Scand 178: 373-383, 2003 .

2. Van Hees AM, Jans A, Hul GB, Roche HM, Saris WH and Blaak EE: Skeletal muscle fatty acid handling in insulin resistant men. Obesity 19: 1350-1359, 2011.

3. Hegarty BD, Cooney GJ, Kraegen EW and Furler SM: Increased efficiency of fatty acid uptake contributes to lipid accumulation in skeletal muscle of high fat-fed insulin-resistant rats. Diabetes 51: 1477-1484, 2002.

4. Koves TR, Ussher JR, Noland RC, et al: Mitochondrial overload and incomplete fatty acid oxidation contribute to skeletal muscle insulin resistance. Cell Metab 7: 45-56, 2008

5. Bonnard C, Durand A, Peyrol S, et al: Mitochondrial dysfunction results from oxidative stress in the skeletal muscle of diet-induced insulin-resistant mice. J Clin Invest 118: 789-800, 2008.

6. Lewis BP, Shih IH, Jones-Rhoades MW, Bartel DP and Burge CB: Prediction of mammalian microRNA targets. Cell 115: 787-798, 2003. 
7. Stefani G and Slack FJ: Small non-coding RNAs in animal development. Nat Rev Mol Cell Biol 9: 219-230, 2008.

8. Lee YS and Dutta A: MicroRNAs in cancer. Annu Rev Pathol 4: 199-227, 2009.

9. Small EM, Frost RJ and Olson EN: MicroRNAs add a new dimension to cardiovascular disease. Circulation 121: 1022-1032, 2010.

10. Kantharidis P, Wang B, Carew RM and Lan HY: Diabetes complications: the microRNA perspective. Diabetes 60 1832-1837, 2011.

11. Pandey AK, Agarwal P, Kaur K and Datta M: MicroRNAs in diabetes: tiny players in big disease. Cell Physiol Biochem 23: 221-232, 2009.

12. Poy MN, Eliasson L, Krutzfeldt J, et al: A pancreatic isletspecific microRNA regulates insulin secretion. Nature 432 : 226-230, 2004

13. He A, Zhu L, Gupta N, Chang Y and Fang F: Overexpression of micro ribonucleic acid 29, highly up-regulated in diabetic rats, leads to insulin resistance in 3T3-L1 adipocytes. Mol Endocrinol 21: 2785-2794, 2007.

14. Ling HY, Ou HS, Feng SD, et al: Changes in microRNA profile and effects of miR-320 in insulin-resistant 3T3-L1 adipocytes. Clin Exp Pharmacol Physiol 36: e32-e39, 2009.

15. Trajkovski M, Hausser J, Soutschek J, et al: MicroRNAs 103 and 107 regulate insulin sensitivity. Nature 474: 649-653, 2011.

16. Poy MN, Hausser J, Trajkovski M, et al: miR-375 maintains normal pancreatic alpha- and beta-cell mass. Proc Natl Acad Sci USA 106: 5813-5818, 2009

17. Chen JF, Mandel EM, Thomson JM, et al: The role of microRNA-1 and microRNA-133 in skeletal muscle proliferation and differentiation. Nat Genet 38: 228-233, 2006.

18. Rosenberg MI, Georges SA, Asawachaicharn A, Analau E and Tapscott SJ: MyoD inhibits Fstl1 and Utrn expression by inducing transcription of miR-206. J Cell Biol 175: 77-85, 2006.

19. Crist CG, Montarras D, Pallafacchina G, Rocancourt D, Cumano A, Conway SJ and Buckingham M: Muscle stem cell behavior is modified by microRNA-27 regulation of Pax3 expression. Proc Natl Acad Sci USA 106: 13383-13387, 2009.

20. Huang B, Qin W, Zhao B, et al: MicroRNA expression profiling in diabetic GK rat model. Acta Biochim Biophys Sin (Shanghai) 41: 472-477, 2009.
21. Herrera BM, Lockstone HE, Taylor JM, et al: Global microRNA expression profiles in insulin target tissues in a spontaneous rat model of type 2 diabetes. Diabetologia 53: 1099-10109, 2010.

22. Gallagher IJ, Scheele C, Keller P, et al: Integration of microRNA changes in vivo identifies novel molecular features of muscle insulin resistance in type 2 diabetes. Genome Med 2: 9, 2010.

23. Karolina DS, Armugam A, Tavintharan S, et al: MicroRNA 144 impairs insulin signaling by inhibiting the expression of insulin receptor substrate 1 in type 2 diabetes mellitus. PLoS One 6: e22839, 2011.

24. Livak KJ and Schmittgen TD: Analysis of relative gene expression data using real-time quantitative PCR and the 2(-Delta Delta C(T)) Method. Methods 25: 402-408, 2001

25. Wang X: miRDB: a microRNA target prediction and functional annotation database with a wiki interface. RNA 14: 1012-1017, 2008.

26. Huang W, Sherman BT, Tan Q, et al: DAVID Bioinformatics Resources: expanded annotation database and novel algorithms to better extract biology from large gene lists. Nucleic Acids Res 35: W169-W175, 2007.

27. McCarthy JJ and Esser KA: MicroRNA-1 and microRNA-133a expression are decreased during skeletal muscle hypertrophy. J Appl Physiol 102: 306-313, 2007.

28. Anderson C, Catoe $\mathrm{H}$ and Werner R: MIR-206 regulates connexin43 expression during skeletal muscle development. Nucleic Acids Res 34: 5863-5871, 2006.

29. Newman JM, Dwyer RM, St-Pierre P, Richards SM, Clark MG and Rattigan S: Decreased microvascular vasomotion and myogenic response in rat skeletal muscle in association with acute insulin resistance. J Physiol 587: 2579-2588, 2009.

30. Gehart H, Kumpf S, Ittner A and Ricci R: MAPK signalling in cellular metabolism: stress or wellness? EMBO Rep 11: 834-840, 2010.

31. Woods YL, Petrie JR and Sutherland C: Dissecting insulin signaling pathways: individualised therapeutic targets for diagnosis and treatment of insulin resistant states. Endocr Metab Immune Disord Drug Targets 9: 187-198, 2009. 